\title{
Antagonist muscle co-activation during straight walking and its relation to kinematics: Insight from young, elderly and Parkinson's disease
}

\author{
Pablo Arias $^{\text {a }, \text { Nelson Espinosa }}{ }^{\text {a }}$, Verónica Robles-García ${ }^{\text {a }}$ Ricardo Cao ${ }^{\mathrm{b}}$, Javier \\ Cudeiro ${ }^{\mathrm{a}}$, \\ ${ }^{a}$ Neuroscience and Motor Control Group (NEUROcom), Department of Medicine-INEF Galicia, Institute of \\ Biomedical Research of A Coruña (INIBIC), Spain \\ bstatistical Inference Research Group (MODES), Department of Mathematics, University of A Coruña, Spain
}

\begin{abstract}
Increased antagonist muscle co-activation of the lower limb during walking seems to be an adaptive process to the physiological changes of aging, in order to gain joint stability. In the healthy subjects this view seems to be reinforced by the fact that the co-activation index (CAI) increases when the gait is faster. The few reports on antagonist coactivation in Parkinson's disease (PD) patients indicate that they have larger co-activation than the healthy elderly, supporting the idea of the stabilization role of CAI during gait, as postural instability is a cardinal feature of PD. However it has also been reported that there is a reduction of the CAI when increasing velocity in PD or normal elderly. This questions the role of coactivation in stabilization during increased velocity. In this study we have analyzed the gait of healthy subjects (young and elderly), and PD patients (with and without freezing of gait, FOG) in order to better understand the relation between co-activation and gait kinematics, and to gain insight into the pathological changes associated with FOG in PD. We used Multiple Linear Regression models to study the relationship in shank muscles between CAI, velocity and cadence. Our results indicate that, for all groups of interest, the relationship between co-activation and the kinematics of gait is poor, due to the high degree of variability, questioning the explanatory value of the index.
\end{abstract}

Keywords: Kinematics. Parkinson. CAI. Multiple Linear Regression. Gait

\section{Introduction}

Muscle co-activation has been studied for a better understanding of motor control of gait. From a theoretical point of view it seems that antagonist muscles in the lower limb should be activated sequentially, avoiding interference in their functions. However it is not clear whether this pattern represents the actual sequence of activation, even in healthy humans. In fact, an increase in antagonist coactivation in the shank and thigh muscles, as a function of the gait velocity, has been reported (Hortobágyi et al., 2009; Schmitz et al., 2009). This suggests an adaptive process aimed at allowing greater stabilization around the joint (Peterson and Martin, 2010; Schmitz et al., 2009). This seems to be supported by the fact that aging can lead to loss of balance (Woollacott, 1993) and stability in gait (Hausdorff, 2007; Hausdorff et al., 2001). Therefore, the larger amount of co-activation displayed by the elderly for a given velocity might contribute to further increase the stability. However, this explanation has been recently challenged since the relationship between aging and increased co-activation of antagonist calf muscles has not been confirmed (Peterson and Martin, 2010). Insight on the role of antagonist muscle co-activation in gait can be gained by evaluating other populations with postural instability, such as people suffering from Parkinson's disease, but very few studies have evaluated this feature in PD during gait (Dietz et al., 1995).

Experiments investigating EMG activity during gait in PD have provided intriguing results. In this case, co-activation in PD seems to follow a different pattern to the one mentioned before, and a reduction in the co-activation index while increasing velocity has been reported (it has also been obtained in healthy 
subjects when walking on a treadmill, Dietz et al., 1995). Interestingly, the study also indicated greater co-activation in the patient than in the healthy group for a given velocity, probably reflecting the above mentioned strategy to increase stability around the joint. It seems that a better understanding on the role of co-activation in gait in these two populations may be obtained by further analyzing different sub-types of subjects, for instance PD with freezing of gait (FOG). EMG analysis from patients suffering FOG revealed an alteration in the timing of discharge of antagonist muscles, tibialis anterior (TA) and gastrocnemius (GA), in the strides prior to the FOG episode (Nieuwboer et al., 2004).

On the other hand, we reasoned that the evaluation of antagonist co-activation can be better achieved employing several variables instead of using only gait velocity. Velocity is just a relation between step/stride cadence and step/stride length which means that a given velocity can be obtained with a whole range of cadence/step length patterns, and so questioning if the co-activation index can be a useful parameter to characterize a given physiological or pathological gait pattern, if only related to one gait variable.

This study is aimed to characterize the co-activation index in gait by examining its relationship to gait velocity and step cadence. We have evaluated different patterns of gait: physiological gait (young and elderly subjects), and pathological gait (advanced PD during ON-dose, and OFF-dose; with and without FOG). The group of patients, with marked postural instability during gait, was included because increasing CAI seems an adaptive process to gain joint stability while walking (Peterson and Martin, 2010; Schmitz et al., 2009). Subjects were asked to walk in synchrony to a metronome beat. This approach has been useful to modulate a whole range of velocities, step lengths, and step cadences in a previous study (Arias and Cudeiro, 2008).

\section{Results}

\subsection{Characterization}

Trochanteral height was comparable across groups, $\mathrm{F}(2,44)=0.376 \mathrm{p}=0.689$; there was however a main effect of age when comparing the groups $\mathrm{F}(2,44)=132.825 \mathrm{p}<0.001$; as expected post-hoc analysis showed that age of Young Control was significantly lower than that of Elderly and $\mathrm{PD}(\mathrm{p}<0.001$ in both cases); but Elderly Controls and PD were comparable in age $(\mathrm{p}=0.449)$. During preferred walking, CAI for the Young Controls was $0.32 \pm 0.04$ (mean \pm SD), for the Elderly Controls was $0.41 \pm 0.06$ and for the $\mathrm{PD}-\mathrm{FOG}_{\mathrm{ON}}$ group was $0.34 \pm 0.04$. When walking as fast as posible the Young Controls were at $0.34 \pm 0.06$, the Elderly Controls $0.42 \pm 0.06$ and the PD group $0.33 \pm 0.04$. Due to safety concerns, the "walking as fast as possible" condition was avoided under OFF conditions and PD+FOG; for these groups during confort gait CAI was $0.35 \pm 0.09$ for the $\mathrm{PD}-\mathrm{FOG}_{\mathrm{OFF}}, 0.31 \pm 0.05$ for the $\mathrm{PD}+\mathrm{FOG}$ an $0.27 \pm 0.04$ for the $\mathrm{PD}+\mathrm{FOG}_{\mathrm{OFF}}$.

\subsection{CAI is not influenced by walking in the presence of a metronome}

Firstly, one-way ANOVA showed the same behavior in all groups when compared with the $\mathrm{CAI}_{\text {stimulated-unstimulated }}$ for a given cadence $\mathrm{F}(5,56)=1.80 \mathrm{p}=0.128$; for a given step length $\mathrm{F}(5,45)=0.40$ $\mathrm{p}=0.848$; and for a given velocity $\mathrm{F}(5,41)=1.72 \mathrm{p}=0.150$. The lack of main effect of factor group in this analysis proved that the behavior was identical in all groups, therefore subsequent analysis was performed with all groups pooled. Subsequently our next approach was to seek if $\mathrm{CAI}_{\text {stimulated-unstimulated was not }}$ significantly different from 0 . The Student t-test for one simple showed that $\mathrm{CAI}_{\text {stimulated-unstimulated }}$ was not significantly different from zero for all the variables analyzed, velocity $t(46)=0.486 p=0.629$, step length $\mathrm{t}(50)=1.090 \mathrm{p}=0.281$; and cadence $\mathrm{t}(62)=1.556 \mathrm{p}=0.125$ (Fig. 1). It is worth saying that lack of differences in stimulated vs. un-stimulated condition in the CAI for a given velocity, step length and cadence, is derived from a whole range of step lengths [0.27-1.37 m], cadences [1.04-2.22 steps/s], and velocities [0.44-2.66 m/s]. This reflects that the use of a metronome to modulate cadence, step length and velocity in a wide range does not make the CAI different from the one obtained in the same range in the absence of metronome. 

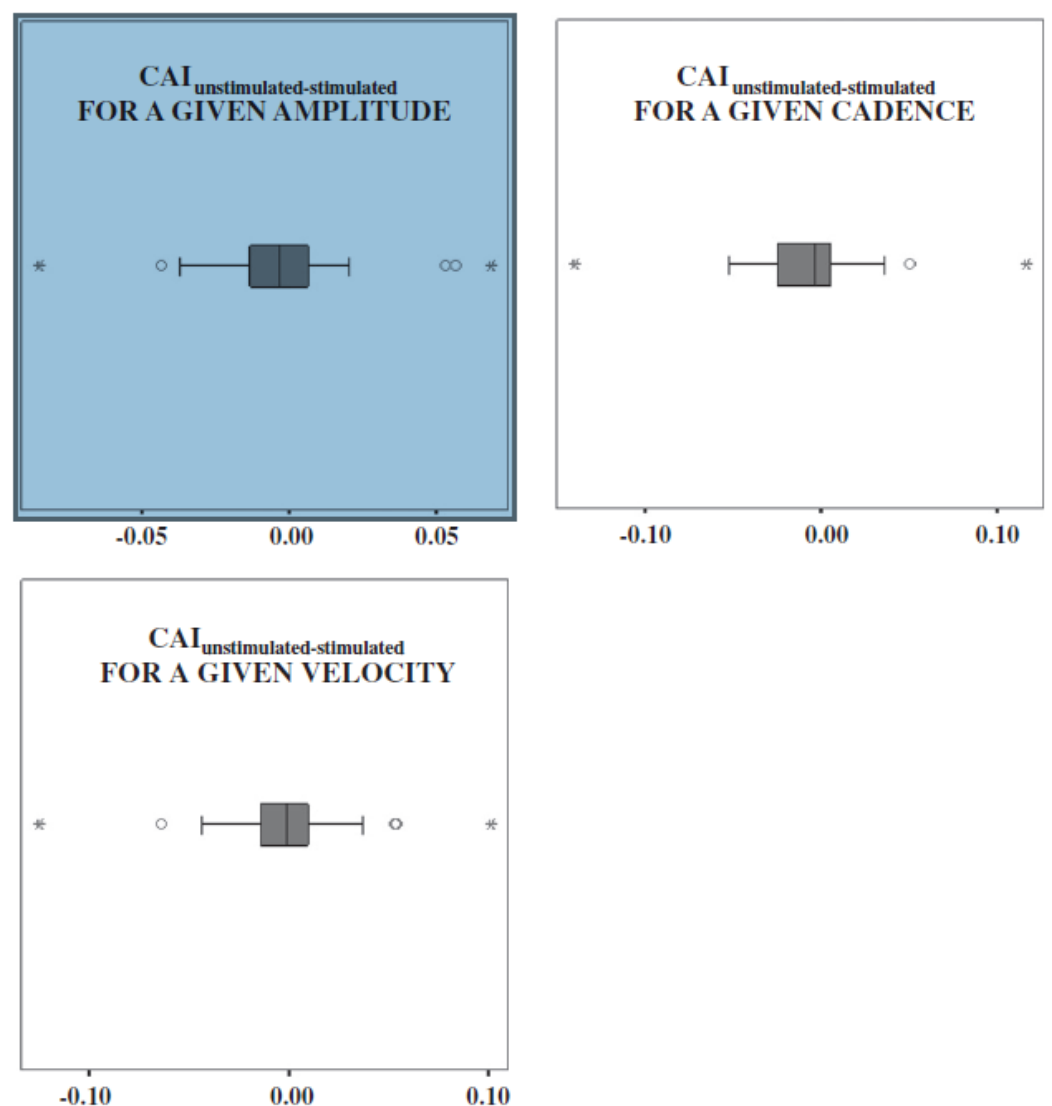

Fig. 1 - The figure represents box and whisker plots for the difference between CAI in the absence and presence of auditory stimulation (the latter used in order to modulate gait), for a given step amplitude (m), a given cadence (steps/s), and a given velocity $(\mathrm{m} / \mathrm{s})$. Dots are outliers (values between 1.5 interquartile ranges (IQRs) and 3 IQRs from the end of a box), and asterisks represent extreme values (more than 3 IQRs from the end of a box). For each un-stimulated amplitude, cadence and velocity, a pair matched stimulated amplitude, cadence and velocity was selected, which was the closest stimulated within $\pm 5 \%$ of the un-stimulated value. For each kinematic variable, once the pair-matched values were identified, their CAI was subtracted $\mathrm{CAI}_{\text {unstimulted - stimuted }}$ which would be close to 0 in the case that the stimulated and un-stimulated conditions were the same. This would prove that the stimulation has no effect on the CAI regardless the gait pattern. Student $t$ test for one sample proved that $\mathrm{CAI}_{\text {unstimulated }- \text { stimulated }}$ was not statistically different from zero for a given amplitude $t(50)=1.090 \mathrm{p}=0.281$, cadence $\mathrm{t}(62)=1.556 \mathrm{p}=0.125$ or velocity $\mathrm{t}(46)=0.486 \mathrm{p}=0.629$, therefore showing that the stimulation has no effect on CAI.

\subsection{CAI and its relation to kinematics}

Then, subsequent MLR analysis was performed to analyze whether CAI depends on velocity and cadence, and whether this relationship is different for different groups (young, elder, PD-FOG ${ }_{\mathrm{ON} \& \mathrm{OFF}}$, and $\left.\mathrm{PD}+\mathrm{FOG}_{\mathrm{ON} \& \mathrm{OFF}}\right)$. For this purpose a total of 353 values were included in the regression analysis.

MLR analysis predicts CAI for $\mathrm{PD}+\mathrm{FOG}_{\mathrm{ON}}=0.402-0.065 \cdot \mathrm{CADENCE} \mathrm{PD}+\mathrm{FOG}_{\mathrm{ON}}+0.031$. VELOCITY $_{\text {PD+FOGON }}+0.006 \cdot \mathrm{CAI}_{\mathrm{YOUNG}}+0.009 \cdot \mathrm{CAI}_{\mathrm{ELDER}}-0.040 \cdot \mathrm{CAI}_{\mathrm{PD}+\mathrm{FOGOFF}}+0.035 \cdot \mathrm{CAI}_{\mathrm{PD}-\mathrm{FOGOFF}}$

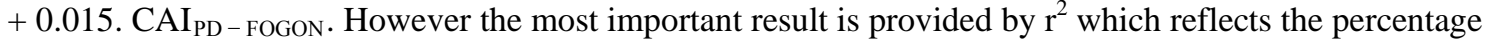
of variability explained by the model. At this point a highly significant $(p<0.001)$ coefficient of determination $\left(r^{2}=0.18\right)$ explains just $18 \%$ of the variability of the model (which reflects the relation of CAI with the different kinematics for the different groups), therefore questioning the validity of CAI during gait for characterizing gait patterns in the young, elder or PD (Figs. 2-4; see also Fig. 5).

The above mentioned model accounts for inter-subject and inter-group variability. To describe intrasubject (trial-to-trial) variability we performed another MLR analysis. The analysis was applied including the factor subject (rather than the factor group). In a first instance the rest of the explanatory variables (i.e. cadence, velocity) were maintained. 


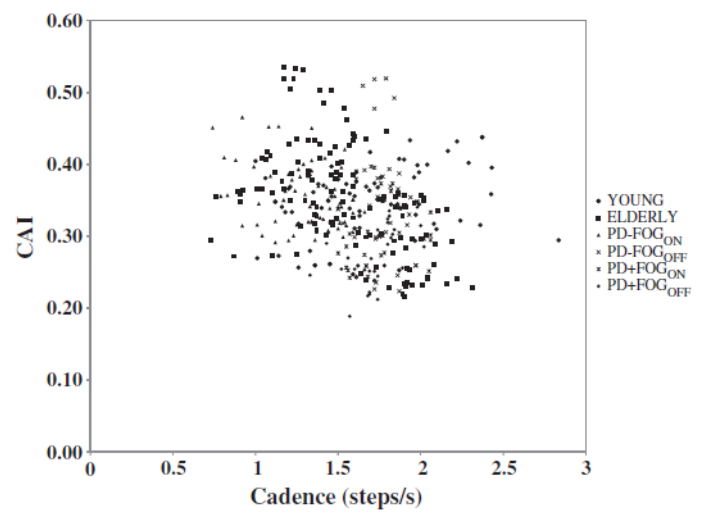

Fig. 2 - The figure represents the relation of one of the kinematic variables (cadence) included in the MLR analysis with CAI. Results from the MLR analysis proved that just $18 \%$ of the variability can be explained by the model, some other approaches different to linear relations have been also used. These results therefore mean that CAI cannot be successfully explained by cadence (or velocity - see Fig. 3), which can easily be seen in the plot. This was observed in all groups of interest (young, elder, and PD), since the MLR results showed identical behavior in all groups. This, therefore, questions the validity of CAI being explained just by the kinematics in order to characterize gait in PD, elderly, or young subjects.

This way the variability explained by the model increases $\left(r^{2}=0.82 ; p<0.001\right)$. However the increment in the predictive value of the model is not due to the relation between the kinematics (velocity and cadence) and CAI. A subsequent exclusión of the kinematics as explanatory variables barely reduces the predictive value $\left(r^{2}=0.78 ; p<0.001\right)$ of the model. The equations were left out to increase clarity (as they include more than 48 and 46 independent variables). The results reinforce the poor relation between the kinematics and CAI and show that every subject has a quite defined CAI poorly affected by the different gait patterns performed.
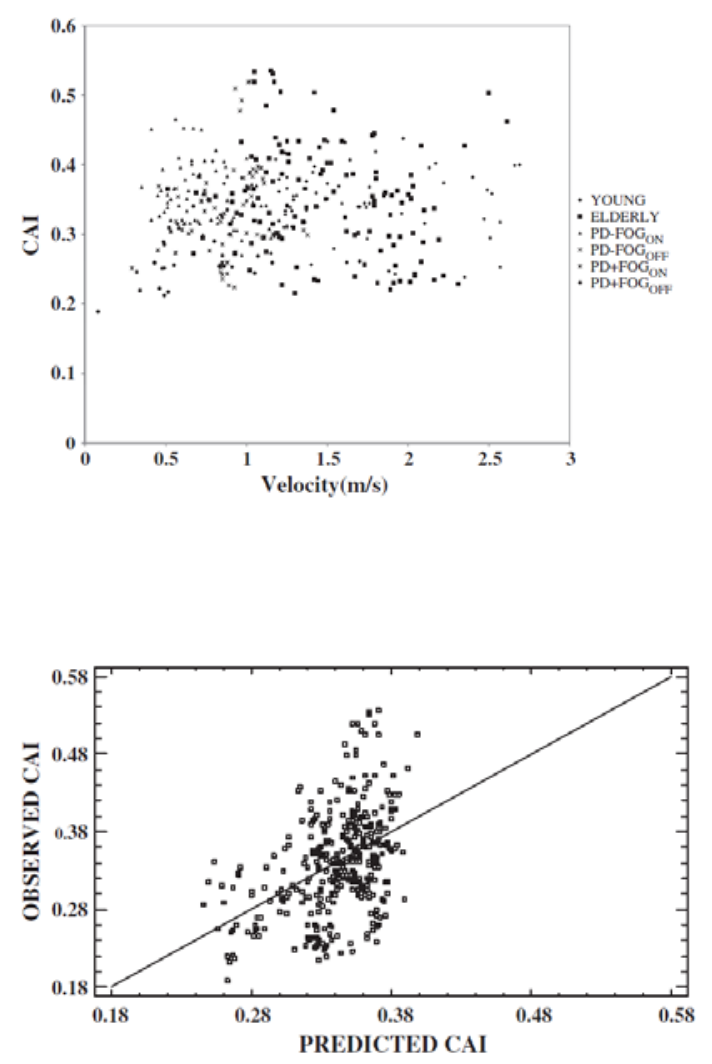

Fig. 3 - The figure represents the relation of another kinematic variable (velocity) included in the MLR analysis with CAI. The same trend as explained in the legend of Fig. 2 for the relation cadence vs. CAI is observed for the relation velocity vs. CAI.

Fig. 4 - This figure depicts the predicted values of CAI using the multivariate linear fit versus the observed CAI values. The diagonal straight line accounts for perfect prediction. As it can be clearly observed in the figure, the dispersion of the point cloud around the diagonal line is large. Thus, the amount of variability of CAI that can be explained by the linear model is low (just $18 \%$, according to $\mathrm{r}^{2}=0.18$ ). 

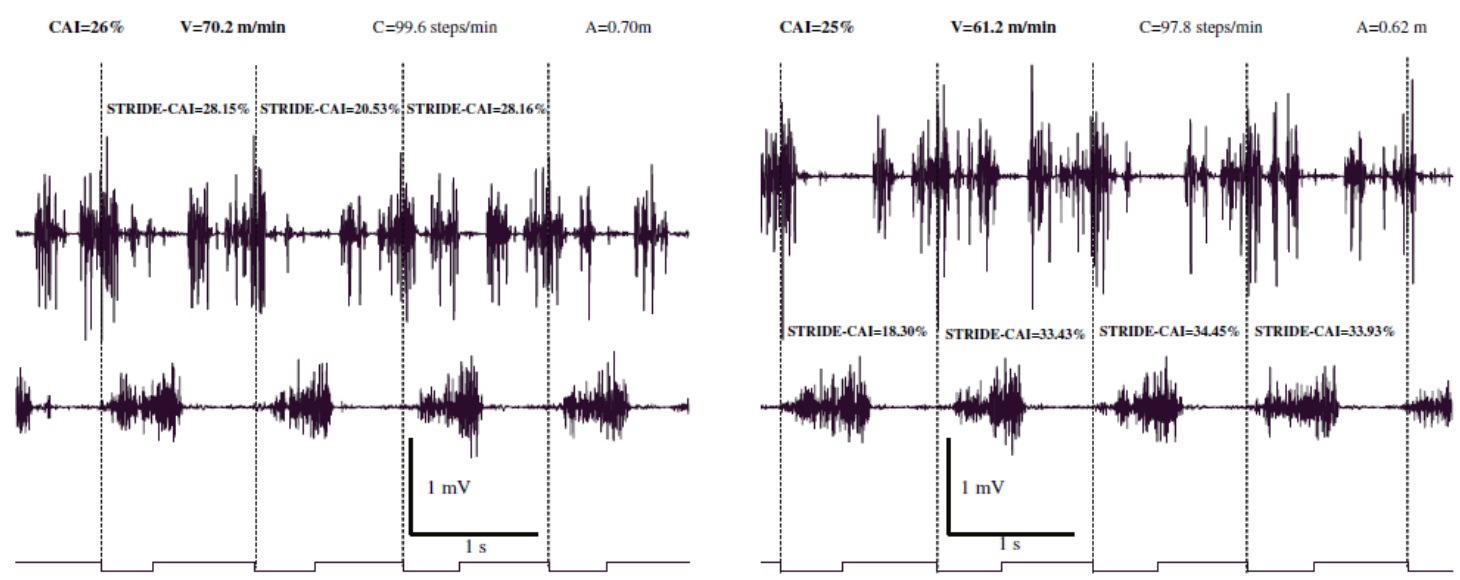

Fig. 5 - The figure represents a sequence of strides of an elder control separated by vertical dotted lines with their CAI. Strides were identified by means of footswitches (line at the bottom). Raw EMG activity is represented with an offset to clearly identify both upper (TI) and lower (SO) muscles. Kinematics (C-cadence, A-amplitude, V-velocity) and CAI at the upper part of the graph are mean values for two different recordings on the same subject. This figure further explains the poor relation between the CAI and the kinematics. Equivalent levels of the CAI (26\% and 25\%) are obtained with different velocities (70.2 and $61.2 \mathrm{~m} / \mathrm{min})$. This clearly reinforces results from Fig. 3 that represent high variability in the relationship between CAI and kinematics.

\section{Discussion}

This works shows that antagonist muscle co-activation of TI and SO during straight gait patterns in different sets of subjects, displaying physiological (young and elderly) and pathological (parkinsonian) gait, presents high variability, not successfully explained by kinematics.

The analysis of antagonist CAI during gait has been chiefly focused on the study of physiological aging (Hortobágyi et al., 2009; Peterson and Martin, 2010; Schmitz et al., 2009) and on some pathologies like stroke (Lamontagne et al., 2000), or knee osteoarthritis (Hubley-Kozey et al., 2009). Some of the above mentioned studies, and others (for example, Mian et al., 2006), evaluated CAI on thigh muscles. While the evaluation of thigh muscles is reasonable for the study of knee pathologies (Hubley-Kozey et al., 2009), it excludes shank muscles (with no direct role on knee functioning, i.e. tibialis anterior). Conversely, we have focused on distal muscles, at which specific EMG-profiles have been shown in subjects with marked postural instability (PD; and in advance to FOG, Nieuwboer et al., 2004).

In the case of elderly some studies have provided conflicting results when trying to relate physiological aging to an increase in the CAI, and increments and decrements in the CAI in shank muscles when increasing velocity have been reported (Dietz et al., 1995; Hortobágyi et al., 2009; Peterson and Martin, 2010). One basic element to understand the increase in CAI when increasing velocity is to gain joint stability (Schmitz et al., 2009), and therefore aging, which is related to impairment in balance (Woollacott, 1993), would further increase the need for larger CAI. We meant to understand this point by reviewing literature on diseases impairing balance, like PD, but the relation between gait and antagonist CAI became further complex. Dietz et al. (1995) reports greater CAI in shank muscles for PD than for healthy, which can be deemed as adaptive processes in order to gain joint stability when walking. However the same work shows both PD and healthy to reduce CAI when increasing velocity (Dietz et al., 1995).

The topic is of interest because if a clear relationship between antagonist CAI and gait patterns can be elucidated it would help to explain gait disturbances in pathological population. For instance, FOG in PD has been reported to be advanced by an altered discharge of antagonist shank muscles while walking (Nieuwboer et al., 2004); therefore determining if CAI in PD freezers presents a characteristic profile might help to differentially advance the prognosis of gait-related disturbances in the disease.

However, when conceiving our study, we clearly thought that the relation of CAI to gait should not only be explained by its simple relation to velocity; it is clear that a given velocity can be achieved with a whole range of different gait patterns (for instance combinations of different patterns of cadence and step length). Therefore when we analyzed CAI in the different populations in relation to velocity and cadence, a more clear depiction of the topic emerged in all populations. Our results comparing subject and groups explain that a given CAI can be displayed with many gait patterns; as the analysis clearly shows that CAI 
presents a high level of variability very poorly explained by cadence, and velocity in all the groups studied. Therefore data from different studies, which could be thought as difficult to match - for instance reduction in CAI when increasing velocity in PD and healthy (Dietz et al., 1995) vs. increase in CAI when increasing velocity in elderly - can just simply be the result of the variability of that relation. On the other hand, variability in the gait related EMG activity is not a new issue: inter-individual variability (Shiavi et al., 1981), or inter-group variability specifically in the groups of interest of the present study (Miller et al., 1996), has been demonstrated. Our results showed no differential effect for the level of variability in the relation CAI vs. any of the kinematics for the different groups; so that no defined association can be established between CAI and kinematics for any group. Intra-individual variability includes stride-to-stride variability (Burden et al., 2003), which reflects different CAIs for subsequent strides (see Fig. 5 for individual stride-CAIs), and also trial-to-trial CAI variability. While evaluation of stride-to-stride-CAI in relation to kinematics would need both stride-to-stride velocity and stride-to-stride cadence (or amplitude), trial-to-trial variability of CAI vs. kinematics is explained by our second MLR. This shows a defined pattern of CAI for each of the different subjects (accounting for nearly 80\% of variability of CAI), with a very small value added if related to the kinematics (velocity and cadence). Then, clearly, the physiological meaning of CAI variability is an issue which must be further studied, since while EMG gait-related variability, in the temporal (and amplitude) domains, might be associated with postural instability, it might also be tied to feed-back adaptability, allowing steady onwards motion (Miller et al., 1996). Our study, then, provides insight on an issue (the variability of CAI in relation to kinematics) which might in previous studies have been misinterpreted or simply passed unnoticed. Possibly, in the future, studies controlling the 3 variables included in the relation "Velocity=Stride Length $\times$ Stride Cadence" would help to show a more clear profile of CAI in its relation to kinematics in subjects with different levels of postural instability - cued-treadmill walking would allow this design. This proposal will also allow evaluation of stride-to-stride CAI variability in relation to stride-to-stride velocity, cadence and amplitude variability (since all of them would be controlled). Notwithstanding, it is clear that results from this study do not question the results for other pathologies at which a more defined pattern seems to have been described, for instance in knee osteoarthritis (Hubley-Kozey et al., 2009) or stroke (Lamontagne et al., 2000).

In conclusion, antagonist CAI in healthy controls and PD during gait gives rise to high variability in relation to the kinematic profile such that, to date, it does not clearly explain physiological or pathological gait profiles in these populations; further designs are needed in order to clarify the relation between CAI and kinematics.

\section{Experimental procedures}

\subsection{Subjects}

47 subjects were recruited for this study; 7 young; 20 elderly; 20 PD in stages III-IV of the Hoehn and Yahr scale (Hoehn and Yahr, 1967). Subjects presented lack of un-correctable auditory-visual impairment, lack of musculoskeletal injury, and scored $>24$ points in Mini Mental Examination Score (Folstein et al., 1975) when checking cognitive impairment.

\subsubsection{Young controls}

Young healthy students were recruited for the study. Their trochanteral height was $0.89 \mathrm{~m}( \pm 0.06)$; and their age was 21.86 yrs $( \pm 1.46)$.

\subsubsection{Elderly controls}

Healthy subjects working in our institution (or their relatives) were selected as the Elderly Control group, age 66.60 yrs $( \pm 7.78)$, trochanteral height $0.87 \mathrm{~m}( \pm 0.05)$.

Both control groups of healthy subjects were screened for any possible history of neurological or psychiatric disorder before the experimental session (interview, MMSE, pull-test). All healthy subjects scored 0 in a pull-test (UPDRS) performed prior to the experimental session; none of them was receiving neuroactive drugs during the week prior to the test. 


\subsubsection{Patients}

Patients belonged to the Association Parkinson Galicia and were approached through the association's neurologist. Patients included in this study were 68.25 yrs $( \pm 6.85)$, and their trochanteral height was $0.88 \mathrm{~m}( \pm 0.07)$. Seven out of 16 patients without history of FOGwere evaluated duringON-dose period (i.e. at the moment when antiparkinsonian medication produced its best effect $-\mathrm{PD}-\mathrm{FOG}_{\mathrm{ON}}$ ); and the other 6 while OFF-dose (i.e. in the absence of antiparkinsonian medication, moment at which parkinsonian signs are displayed with greatest severity - PD-FOG ${ }_{\mathrm{OFF}}$ ). All patients with history of FOG were evaluated during both $\mathrm{ON}$ and $\mathrm{OFF}$ doses, $\left(\mathrm{PD}+\mathrm{FOG}_{\mathrm{ON}}\right)$ and $\left(\mathrm{PD}+\mathrm{FOG} \mathrm{OFF}_{\mathrm{OF}}\right)$, respectively.

4.1.3.1. PD+FOG. Patients in this group, who exhibited significant FOG, had to match the following criteria:

- diagnosis of idiopathic PD based on the UK Parkinson's Disease Society Brain Bank for clinical diagnostic criteria (Hughes et al., 1992)

- history of freezing during walking, confirmed from medical records, and score $>10$ (and $\geq 2$ in item \#3) in the Freezing of Gait Questionnaire (FOGQ) (Giladi et al., 2000)

- predictable motor fluctuation related to dose intake, determined from medical records and neurological examination

- at the moment of testing, they should be able to walk $6 \mathrm{~m}$ unaided, turn around and come back, despite the freezing episodes at turning or start walking. Given the nature of this study patients suffering from FOG during the straight part of the task were excluded from the analysis.

4.1.3.2. PD-FOG. PD without history of FOG were also recruited. Inclusion criteria were the same as stated for PD+FOG, with the exception of those criteria related to FOG. The score in the FOGQ had to be zero to be a possible subject in this group.

\subsection{Procedure}

Subjects walked along a straight walkway $6 \mathrm{~m}$ long until $24 \mathrm{~m}$ is completed. Data from turns at the end of the walkway were not analyzed given that our objective was to analyze the relation of co-activation to straight gait parameters.

\subsubsection{Walking conditions}

Initially subjects had to walk at their preferred walking (PW) cadence for the first $24 \mathrm{~m}$. In the next condition subjects were asked to walk as fast as possible (FW). Afterwards, and in order to modulate gait velocity, cadence and step length, subjects were asked to walk matching their steps to a metronome, which was set at different percentages of each subject's fast walking cadence (from 50 to $110 \%$ FWcadence; order of presentation was randomized). This procedure has been shown tomodulate gait patterns in advance PD without freezing, and in healthy controls (Arias and Cudeiro, 2008); a stimulation condition including a frequency matching PW cadence was also included.

In the case of PD+FOG and PD-FOG ${ }_{\mathrm{OFF}}$ the stimulation conditions and their number were modified given the unability shown by the subjects to walk safely in the fast walk condition, and also because of the troublesome gait displayed during the slowest stimulation frequencies. Therefore these patients were asked to walk at their preferred un-stimulated cadence and also in the presence of a metronome 10 and 20\% faster than the PW cadence. Having into account the previous data that shows that auditory stimulation matching PW cadence induces gait patterns with lower cadence tan un-stimulated PW cadence (Arias and Cudeiro, 2008), this condition was included in order to modulate cadence downwards. 12 of the healthy controls performed only this protocol in order to match controls for the different subsets of PD's protocols. The protocol was bound to the Helsinki declaration and approved by our institution's ethics committee; subjects signed consent forms.

\subsection{Material}

\subsubsection{EMG and kinematic recordings}

Biometrics DataLog (Biometrics Ltd, Gwent, UK) was utilized for EMG recordings sampled at 1 $\mathrm{kHz}$; bipolar surface electrodes were placed bilaterally over tibialis anterior (TI) and soleus (SO) muscles following standard procedures (Cram et al., 1998). Probe skin electrodes (Biometrics SX230) included 
preamplifiers (gain 1000) and low/high pass (20-450 Hz) were place over the muscles. Skin preparation included shaving, abrading and cleaning with alcohol; EMG gel was applied over the poles of the electrode in order to reduce impedance to the minimum level. Adhesive pads were fixed to the electrode and then bilaterally to the skin. The ground electrode was placed over the wrist. Contact footswitches were integrated by means of the digital channels of the DataLog System, which was synchronized to the photocells allowing registration of feet contact; sampling rate for each pair of footswitch was sampled at $1 \mathrm{kHz}$. Data was stored for detailed OFF-line analysis using customized programs implemented in MatLab.

\subsection{Data processing}

EMG recordings for each muscle were processed including full-wave rectification and averaging with a sample-time constant of $10 \mathrm{~ms}$, and normalization in amplitude. Age-related sensorimotor impairment leads to activation deficit during MVC (Stevens et al., 2003), and this is maximized in PD due to deficits in kinesthesia (Klockgether et al., 1995) (i.e. PD+FOG ${ }_{\mathrm{OFF}}$ was hardly capable of recruiting soleus muscle during MVC against an external load). Therefore, we normalized EMG activity taking as reference peak amplitude during baseline gait. This is in line with works avoiding the use of maximal voluntary contraction (MVC) for normalizing EMG-activity in elder or PD in different movements, including gait (Caliandro et al., 2011; Murley et al., 2010; Peterson and Martin, 2010; Powell et al., 2011). The coactivation index (CAI)was calculated following the equation described by Winter (1990), for which a minimum of 16 strides were analyzed in each subject, which were identified through footswitches recordings (Fig. 5):

$\mathrm{CAI}=2 \cdot\left(\int \min \{\operatorname{EMGi}(t), \operatorname{EMGj}(t)\} \mathrm{dt} / \int(\operatorname{EMGi}(t) \mathrm{dt}+\operatorname{EMGj}(t) \mathrm{dt})\right)$

The numerator of the equation reflects the amount of overlapping activity (common area) from both muscles EMGi and EMGj (TA and SO), divided by the sum of both areas.

All the 7 PD+FOG displayed freezing during the whole walking task but in all of them FOG appeared when turning or starting walking, which are by far the most frequent types of FOG (Arias and Cudeiro, 2010; Giladi et al., 1992); recordings from turning were not included into the analysis.

\subsection{Analyzed variables}

Variables of interest were:

CAI calculated as mentioned before (see Eq. 1). Given PD displayed bilateral affectation (stage III-IV Hoehn and Yahr), the average of both legs was used, following the same procedure as for healthy.

Velocity calculated from the distance covered and the time (achieved through photocells) needed to complete the distance, expressed in $\mathrm{m} / \mathrm{s}$.

Cadence number of steps/s, calculated from footswitches data.

Step length derived as a function of velocity and cadence, expressed in $\mathrm{m}$.

\subsection{Statistical analysis}

Our first approach to the statistical analysis was to evaluate a possible effect of the auditory stimulation (strategy used to modulate kinematics of gait) on modifying CAI with respect to unstimulated gait in all groups. Thus, for each of the kinematic variables (cadence, step length, and velocity) we matched the un-stimulated condition (PW or FW) to the closest stimulation condition (if any) within a range of $\pm 5 \%$ of the un-stimulated one. Thus, if one subject cadence was $100 \mathrm{steps} / \mathrm{min}$ in one unstimulated condition, it was looked for the closest cadence in the presence of stimulation within the range defined (95-105 steps/min; in this case); the same for step length and velocity. Subsequently, for each pair-matched value the difference in their CAI (CAI in the stimulated condition minus the CAI in the unstimulated condition) was calculated ( $\left.\mathrm{CAI}_{\text {stimulated - unstimulated }}\right)$. Then, to know if this relation depends on group, a one-way ANOVA was performed with Group as main factor for the $\mathrm{CAI}_{\text {stimulated }- \text { unstimulated }}$. Therefore if the group effect is not statistically significant we assume the behavior of all groups to be the same (for each variable). So we performed further statistical analysis with all groups pooled. Subsequently, the $\mathrm{CAI}_{\text {stimulated - unstimulated }}$ was used to know if the auditory stimulation modifies the CAI for a given gait pattern, which in case of not being affected by stimulation will tend to be 0 for each pair 
stimulated/un-stimulated. This was checked by applying a one-sample Student t-test setting 0 as the reference value, therefore lack of statistical difference would mean there is no difference in the stimulated vs. un-stimulated condition for a given value (of cadence, step length, and velocity). Since the test was not significant for all the assumptions lack of effect of stimulation over CAI is confirmed.

Then, in order to characterize CAI gait pattern for each group, and its relation to cadence, step length, and velocity, Multiple Linear Regression (MLR) analysis was performed, setting the CAI as the dependent variable, and the variables Group (young, elder, $\mathrm{PD}-\mathrm{FOG}_{\mathrm{ON}}, \mathrm{PD}-\mathrm{FOG} \mathrm{OFF}_{\mathrm{OF}}, \mathrm{PD}+\mathrm{FOG} \mathrm{ON}_{\mathrm{ON}}$, $\mathrm{PD}+\mathrm{FOG}_{\mathrm{OFF}}$ ), Cadence, and Velocity, as explanatory variables, therefore predicting the relation of CAI to the different kinematics, and explaining the inter-subject and inter-group variability.

Step length was left out of the model given that the included variables must not be directly dependent. Since there exists a direct relation between velocity cadence and step length, and because velocity and cadence were included as explanatory variables, step length was the one left out. This was selected given that the relation between Velocity and CAI is of interest considering the background provided by literature, and Cadence is also of interest since the stimulation proposed aimed to match step cadence.

Subsequently the same model was applied again to evaluate intra-subject (trial to trial) variability. In a first instance for this purpose the dependent variable was the same CAI, and the explanatory variables were Cadence, Velocity, and Subject (rather than Group). Afterwards, the analysis was performed again, but leaving out the kinematic variables as explanatory variables to understand its impact on the CAI.

Data was analyzed using Statgraphics. Normality was assessed by Kolmógorov-Smirnov test, and heteroscedasticity was checked using Durwin-Watson test. Significance was set at 0.05 .

\section{Acknowledgments}

This work was supported by Xunta de Galicia (Consellería de Educación-2007/000140-0 and Dirección Xeral de I+D+i; 2010-2012), Spain. We are thankful to the Asociación Parkinson Galicia. We are indebted to Dr Kenneth L. Grieve for his helpful advice and corrections in the manuscript.

\section{REFERENCES}

Arias, P., Cudeiro, J., 2008. Effects of rhythmic sensory stimulation (auditory, visual) on gait in Parkinson's disease patients. Exp. Brain Res. 186, 589-601.

Arias, P., Cudeiro, J., 2010. Effect of rhythmic auditory stimulation on gait in Parkinsonian patients with and without freezing of gait. PLoS One 5, e9675.

Burden, A.M., Trew, M., Baltzopoulos, V., 2003. Normalisation of gait EMGs: a re-examination. J. Electromyogr. Kinesiol. 13, 519-532.

Caliandro, P., Ferrarin, M., Cioni, M., Bentivoglio, A.R., Minciotti, I., D'Urso, P.I., Tonali, P.A., Padua, L., 2011. Levodopa effect on electromyographic activation patterns of tibialis anterior muscle during walking in Parkinson's disease. Gait Posture 33, 436-441.

Cram, J.R., Kasman, G.S., Holtz, J., 1998. Introduction to Surface Electromyography. Aspen Publishers. Vol.,.Dietz, V., Zijlstra, W., Prokop, T., Berger, W., 1995. Leg muscle activation during gait in Parkinson's disease: adaptation and interlimb coordination. Electromyog. Motor Control-Electroencephalog. Clin. Neurophysiol. 97, 408-415.

Folstein, M.F., Folstein, S.E., McHugh, P.R., 1975. Mini-mental state. A practical method for grading the cognitive state of patients for the clinician. J. Psychiatr. Res. 12, 189-198.

Giladi, N., McMahon, D., Przedborski, S., Flaster, E., Guillory, S., Kostic, V., Fahn, S., 1992. Motor blocks in Parkinson's disease. Neurology 42, 333-339.

Giladi, N., Shabtai, H., Simon, E.S., Biran, S., Tal, J., Korczyn, A.D., 2000. Construction of freezing of gait questionnaire for patients with Parkinsonism. Parkinsonism Relat. Disord. 6, 165-170.

Hausdorff, J.M., 2007. Gait dynamics, fractals and falls: finding meaning in the stride-to-stride fluctuations of human walking. Hum. Mov. Sci. 26, 555-589.

Hausdorff, J.M., Ashkenazy, Y., Peng, C.K., Ivanov, P.C., Stanley, H.E., Goldberger, A.L., 2001. When human walking becomes random walking: fractal analysis and modeling of gait rhythm fluctuations. Physica A 302, 138-147.

Hoehn, M.M., Yahr, M.D., 1967. Parkinsonism — onset, progression, and mortality. Neurology 17, 427-442.

Hortobágyi, T., Solnik, S., Gruber, A., Rider, P., Steinweg, K., Helseth, J., DeVita, P., 2009. Interaction between age and gait velocity in the amplitude and timing of antagonist muscle coactivation. Gait Posture 29, 558-564. 
Hubley-Kozey, C.L., Hill, N.A., Rutherford, D.J., Dunbar, M.J., Stanish, W.D., 2009. Co-activation differences in lower limb muscles between asymptomatic controls and those with varying degrees of knee osteoarthritis during walking. Clin. Biomech. 24, 407-414 Bristol, Avon.

Hughes, A.J., Daniel, S.E., Kilford, L., Lees, A.J., 1992. Accuracy of clinical-diagnosis of idiopathic Parkinson's disease - a clinicopathological study of 100 cases. J. Neurol. Neurosurg. Psychiatry 55, 181-184.

Klockgether, T., Borutta, M., Rapp, H., Spieker, S., Dichgans, J., 1995. A defect of kinesthesia in Parkinson's disease. Mov. Disord. 10, 460-465.

Lamontagne, A., Richards, C.L., Malouin, F., 2000. Coactivation during gait as an adaptive behavior after stroke. J. Electromyogr. Kinesiol. 10, 407-415.

Mian, O.S., Thom, J.M., Ardigo, L.P., Narici, M.V., Minetti, A.E., 2006.

Metabolic cost, mechanical work, and efficiency during walking in young and older men. Acta Physiol. (Oxf). 186, 127-139.

Miller, R.A., Thaut, M.H., McIntosh, G.C., Rice, R.R., 1996

Components of EMG symmetry and variability in parkinsonian and healthy elderly gait. Electroencephalogr. Clin. Neurophysiol. 101, 1-7.

Murley, G.S., Menz, H.B., Landorf, K.B., Bird, A.R., 2010. Reliability of lower limb electromyography during overground walking: a comparison of maximal- and sub-maximal normalisation techniques. J. Biomech. 43, 749756.

Nieuwboer, A., Dom, R., De Weerdt, W., Desloovere, K., Janssens, L., Stijn, V., 2004. Electromyographic profiles of gait prior to onset of freezing episodes in patients with Parkinson's disease. Brain 127, 1650-1660.

Peterson, D.S., Martin, P.E., 2010. Effects of age and walking speed on coactivation and cost of walking in healthy adults. Gait Posture 31, 355-359.

Powell,D., JosephThrelkeld, A., Fang, X.,Muthumani,A., Xia, R., 2011. Amplitude- and velocity-dependency of rigiditymeasured at the wrist in Parkinson's disease. Clin. Neurophysiol.

Schmitz, A., Silder, A., Heiderscheit, B., Mahoney, J., Thelen, D.G., 2009. Differences in lower-extremity muscular activation during walking between healthy older and young adults. J. Electromyogr. Kinesiol. 19, 1085-1091.

Shiavi, R., Champion, S., Freeman, F., Griffin, P., 1981. Variability of electromyographic patterns for level-surface walking through a range of self-selected speeds. Bull. Prosthet. Res. 10-35, 5-14.

Stevens, J.E., Stackhouse, S.K., Binder-Macleod, S.A., Snyder-Mackler, L., 2003. Are voluntary muscle activation deficits in older adults meaningful? Muscle Nerve 27, 99-101.

Winter, D.A., 1990. Biomechanics and Motor Control of Human Movement. John Wiley Edt. Vol.

Woollacott, M.H., 1993. Age-related-changes in posture and movement. J. Gerontol. 48, 56-60 